\section{Una dieta antiinflamatoria disminuiría el riesgo de mortalidad por todas las causas}

\section{An anti-inflammatory diet is associated with lower mortality risk from all causes}

\section{Señor Editor,}

Los procesos inflamatorios están involucrados en una gran variedad de problemas de salud, siendo actualmente la inflamación crónica sistémica (ICS) la principal causa de discapacidad y mortalidad a nivel mundial ${ }^{1}$. El 50\% de todas las muertes son atribuibles a enfermedades relacionadas con la inflamación, tales como enfermedades cardiovasculares (ECV), cáncer, diabetes mellitus tipo 2, enfermedad renal crónica, enfermedad de hígado graso no alcohólico, además de enfermedades autoinmunes y neurodegenerativas ${ }^{1}$. Entre los factores que promueven la ICS se destaca el perfil alimentario actual de la población, caracterizado por un mayor consumo de alimentos ultra procesados, altos en cereales refinados, sal, grasas trans y aditivos, junto a un alto consumo de alcohol y bajo en frutas, verduras y alimentos ricos en fibra dietética. Consecuentemente, disminuir el consumo de alimentos proinflamatorios y aumentar el consumo de alimentos saludables y antiinflamatorios, como frutas, verduras de hojas verdes, sería clave en la reducción de estas enfermedades y la mortalidad asociada ${ }^{1,2}$. En la Tabla 1 se resumen aquellos alimentos denominados proinflamatorios, que son consumidos en mayor proporción en el patrón dietético occidental vs aquellos alimentos antiinflamatorios cuyo consumo debería privilegiarse, caracterizando un patrón de alimentación saludable ${ }^{3-5}$.

$\mathrm{El}$ análisis de patrones dietéticos ha resultado ser más predictivo que el análisis del consumo de alimentos y nutrientes en forma asiladas al momento de examinar la relación entre la dieta y el riesgo de enfermedades crónicas ${ }^{6}$. Existen estudios en los cuales, a través del seguimiento a diversas poblaciones, han logrado crear índices o puntuaciones de calidad dietética, considerando múltiples factores alimentarios para evaluar de manera integral la asociación de algunos patrones dietéticos y el riesgo de enfermedades vinculadas a la ICS ${ }^{4-6}$.

Un ejemplo es el estudio publicado en la revista Journal of Internal Medicine $e^{4}$ que reportó la asociación entre el cumplimiento de una dieta con alto potencial antiinflamatorio con mayor tiempo de supervivencia y menor mortalidad por todas las causas, por ECV y por cáncer. Este estudio prospectivo evaluó dos cohortes, con un total de 68.273 hombres y mujeres de origen sueco (45-83 años), a quienes se les realizó un seguimiento durante 16 años, obteniéndose información acerca de datos sociodemográficos, antropométricos, estilos de vida, diagnóstico de enfermedades y causa de muerte. Utilizando 16 alimentos (11 con potencial antiinflamatorio y 5 con potencial proinflamatorio), los autores crearon un índice para identificar el nivel de adherencia a la dieta antiinflamatoria. Dentro de sus resultados se observó que los participantes del cuartil más alto del índice de dieta antiinflamatoria, mostraron $18 \%$ menor riesgo de mortalidad en total,, $20 \%$ menor riesgo de muerte por ECV y un riesgo $13 \%$ menor de mortalidad debido al cáncer, comparados con aquellos del cuartil más bajo. Además, los autores reportaron un mayor beneficio de la dieta antiinflamatoria en los fumadores, evidenciando menor riesgo de mortalidad por las tres causas previamente analizadas ${ }^{4}$.

Por otra parte, la adherencia a una dieta proinflamatoria se ha asociado con un aumento del riesgo de desarrollar cáncer colorrectal. Así lo evidenció un estudio publicado por Tabung y cols. en la revista JAMA Oncology, cuyo objetivo fue caracterizar el potencial inflamatorio de la dieta y dilucidar su papel en el desarrollo del cáncer colorrectal. Los autores usaron un índice asociado al patrón inflamatorio dietético empírico (EDIP), basado en una suma ponderada de 18 grupos de alimentos que caracterizan el potencial inflamatorio en función de los niveles circulantes de biomarcadores de inflamación ${ }^{5}$. El estudio utilizó datos de 2 cohortes de adultos de Estados Unidos: "Nurses Health Study" (NHS), formada por 74.246 mujeres (30-55 años, al comienzo del estudio en 1976), seguidas durante 26 años; y "Health Professionals Follow-up Study" (HPFS), que registró 46.804 hombres (40-75 años, al comienzo del estudio en 1986), seguidos por 24 años. Al comparar los participantes que consumían las dietas más proinflamatorias (quintil más alto del índice EDIP) con aquellos con dietas menos proinflamatorias (quintil más bajo del índice EDIP), se observó un 32\% más de riesgo de desarrollar cáncer colorrectal; al separar por sexo, los hombres tenían $44 \%$ más riesgo, mientras que las mujeres tenían $22 \%$ más de riesgo. El riesgo de desarrollar cáncer colorrectal fue aún mayor entre hombres con sobrepeso u obesidad y mujeres bajo peso, así como también, entre los hombres y mujeres que no consumían alcohol, lo que indica que las estrategias para reducir el papel adverso de un patrón dietético proinflamatorio en el desarrollo del cáncer colorrectal pueden tener mayores beneficios entre estos últimos grupos de personas. Los hallazgos de este estudio prospectivo sugieren que la inflamación es un mecanismo potencial que vincula los patrones dietéticos y desarrollo de cáncer colorrectal ${ }^{5}$.

Considerando que la geografía privilegiada de Chile ofrece la posibilidad de acceder a una gran variedad de alimentos de origen vegetal con propiedades antioxidantes y antiinflamatoria, es fundamental educar a la población acerca de los beneficios de estos productos y la importancia de incorporar alimentos saludables y antiinflamatorios a la dieta como una herramienta 
Tabla 1. Alimentos antiinflamatorios y alimentos proinflamatorios

\begin{tabular}{|ll|}
\hline Alimentos antiinflamatorios & Alimentos proinflamatorios \\
$\begin{array}{l}\text { Verduras de hoja verde (espinacas, kale, acelgas). Verduras } \\
\text { crucíferas (brócoli, repollo, coliflor, coles de Bruselas). Tomate }\end{array}$ & $\begin{array}{l}\text { Carnes rojas sin procesar y procesadas (hamburguesas, file- } \\
\text { tesas, salchichas) }\end{array}$ \\
\hline Frutas (cítricos, berries, manzana, granada) & Papas fritas y otros alimentos fritos \\
\hline Té, café & Carbohidratos refinados (pan blanco y pasteles) \\
Cereales integrales & Bebidas azucaradas y otras bebidas endulzadas con azúcar \\
Aceite de oliva y de canola & Margarinas y manteca de cerdo \\
\hline Frutos secos (nueces, almendras) & \\
\hline Pescados grasos (salmón, atún, sardinas) & \\
\hline Legumbres & \\
\hline
\end{tabular}

Fuente: Adaptado de Zhu y cols.; Kaluza y cols.; Tabung y cols. . $^{3-5}$.

preventiva de enfermedades crónicas no transmisibles, en especial ECV y cáncer, que presentan altas prevalencias y se encuentran dentro de las primeras causas de muerte en nuestro país. Fomentar el consumo y el comercio de alimentos naturales en la población, pudiera ser una estrategia efectiva para el reducir el riesgo de estas patologías.

Solange Parra-Soto ${ }^{1,2, a}$, María Adela MartínezSanguinetti ${ }^{3, b}$, Ana María Leiva-Ordoñez ${ }^{4, c}$, Fanny Petermann-Rocha ${ }^{1,2, d}$, Nicole Lasserre-Laso ${ }^{5, d}$, Carlos Celis-Morales ${ }^{1,2,6, e}$

${ }^{1}$ Institute of Health and Wellbeing, University of Glasgow.Glasgow, United Kingdom. ${ }^{2}$ BHF Glasgow Cardiovascular Research Centre, Institute of Cardiovascular and Medical Sciences, University of Glasgow. Glasgow, United Kingdom. ${ }^{3}$ Instituto de Farmacia, Facultad de Ciencias, Universidad Austral de Chile. Valdivia, Chile. ${ }^{4}$ Instituto de Anatomía, Histología y Patología, Facultad de Medicina, Universidad Austral de Chile. Valdivia, Chile.

${ }^{5}$ Escuela de Nutrición y Dietética, Facultad de Salud, Universidad Santo Tomás, Chile.

${ }^{6}$ Laboratorio de Rendimiento Humano, Grupo de Estudio en Educación, Actividad Física y Salud (GEEAFyS), Universidad Católica del Maule. Talca, Chile.

${ }^{a}$ Nutricionista. MSc. en Nutrición y Alimentos, mención Promoción de la Salud.

${ }^{b}$ Bioquímica. MSc. en Nutrición y Dietética. 'Bióloga. MSc. en Neurociencias y Salud Mental.

${ }^{d}$ Nutricionista. MSc. Nutrición Humana

eProfesor de Educación Física Dr. en Ciencias Cardiovasculares y Biomédicas.

\section{Referencias}

1. Furman D, Campisi J, Verdin E, Carrera-Bastos P, Targ S, Franceschi C, et al. Chronic inflammation in the etiology of disease across the life span. Nat Med 2019; 25 (12): 1822-32. doi: 10.1038/s41591-019-0675-0.

2. Shivappa N, Godos J, Hébert JR, Wirth MD, Piuri G, Speciani AF, et al. Dietary Inflammatory Index and Cardiovascular Risk and Mortality-A Meta-Analysis. Nutrients 2018; 10 (2): 200. doi:10.3390/nu10020200.

3. Zhu F, Du B, Xu B. Anti-inflammatory effects of phytochemicals from fruits, vegetables, and food legumes: A review. Crit Rev Food Sci Nutr 2018; 58 (8): 1260-70.

4. Kaluza J, Håkansson N, Harris HR, Orsini N, Michaëlsson $\mathrm{K}$, Wolk A. Influence of anti-inflammatory diet and smoking on mortality and survival in men and women: two prospective cohort studies. J Intern Med 2019; 285: 75-91.

5. Tabung FK, Liu L, Wang W, Fung TT, Wu K, Smith-Warner SA, et al. Association of Dietary Inflammatory Potential With Colorectal Cancer Risk in Men and Women. JAMA Oncology 2018; 4 (3): 366-73.

6. Hu FB. Dietary pattern analysis: A new direction in nutritional epidemiology. Curr Opin Lipidol 2002; 13 (1): 3-9.

Correspondencia a:

María Adela Martínez-Sanguinetti

Instituto de Farmacia, Facultad de Ciencias, Universidad Austral de

Chile, Valdivia, Chile.

mmartin3@uach.cl 